University of Nebraska - Lincoln

DigitalCommons@University of Nebraska - Lincoln

\title{
The effect of heterogeneity on plane wave propagation through layered composites
}

\author{
X. Chen \\ Florida State University \\ Namas Chandra \\ University of Nebraska-Lincoln, nchandra2@unl.edu
}

Follow this and additional works at: https://digitalcommons.unl.edu/mechengfacpub

Part of the Mechanical Engineering Commons

Chen, $\mathrm{X}$. and Chandra, Namas, "The effect of heterogeneity on plane wave propagation through layered composites" (2004). Mechanical \& Materials Engineering Faculty Publications. 57.

https://digitalcommons.unl.edu/mechengfacpub/57

This Article is brought to you for free and open access by the Mechanical \& Materials Engineering, Department of at DigitalCommons@University of Nebraska - Lincoln. It has been accepted for inclusion in Mechanical \& Materials Engineering Faculty Publications by an authorized administrator of DigitalCommons@University of Nebraska Lincoln. 
Published in Composites Science and Technology 64:10-11 (August 2004), pp. 1477-1493;

doi: 10.1016/j.compscitech.2003.10.024 Copyright @ 12004 X. Chen and N. Chandra;

published by Elsevier Science Ltd. Used by permission.

Submitted July 14, 2003; revised October 20, 2003; accepted October 28, 2003; published online February 7, 2004.

\title{
The effect of heterogeneity on plane wave propagation through layered composites
}

\author{
X. Chen and N. Chandra \\ Department of Mechanical Engineering, College of Engineering, Florida State University, \\ 2525 Pottsdamer Street, Room 229, Tallahassee, FL 32310, USA \\ Corresponding author - N. Chandra
}

\begin{abstract}
When laminated composites are subjected to impact loading, the material response is critically determined by the interactions of multiple waves generated at the laminate interfaces. Due to the high complexity arising from the architectural details of composites, layered heterogeneous materials have been studied as the model system to understand the impact behavior of engineering composites. Previously, the present authors have developed an analytical solution to the problem of plate impact of layered systems; plate impact test is a standard boundary value problem used to study high velocity impact behavior both in the elastic and shock wave regimes. In this paper, we examine the various heterogeneity factors that affect the impact response of the laminated composite systems. We have identified three different heterogeneity factors (impedance mismatch, interface density and thickness ratio) and examine their effects on wave scattering. These effects are then used to explain some outstanding experimental observations in terms of shock wave structure (arrival time, sloping rise, peak stress and oscillatory pulse duration). It is shown that though the results pertain to layered systems, the observations can be qualitatively extended to real composites.
\end{abstract}

Keywords: layered structures, impact behavior, stress wave scattering

\section{Introduction}

The understanding of the behavior of materials under very high strain rate loading conditions is vital in many areas of civilian and military applications. So far, the best practical structures/materials to absorb impact energy and resist impact damage are designed in the form of layered composites. Composite materials with organic matrices reinforced by synthetic or ceramic fibers either individually or in combination with monolithic ceramic layers are being used to achieve light weight and enhanced ballistic impact resistance for body armor and vehicle protection [1-3]. In particular, glassreinforced plastics (GRP) have been well recognized as potential candidates for these applications. In order to characterize the dynamic behavior of materials under impact loading, well-controlled experiments are usually carried out using plate impact tests. Uniaxial strain levels up to extremely high values can be generated at the geometric center of impact plane. The material response under plate impact tests is acquired in the form of either stress vs. time or velocity vs. time data. These time-resolved measurement techniques yield wave profiles with detailed structure that provides a wealth of information about the shock response of the material. As a result, there is growing research interest in understanding the response of composite materials subjected to plate impact loadings.

Up to now, partly due to the diversity and complexity of the composites, there is a paucity of controlled experiments on laminated composites subjected to high velocity impact loadings. Though laminated composites exhibit spatial variations in geometry and material properties, it is often true that these variations are quasiperiodic. So, when modeling wave propagation in laminated composites, researchers have used idealized pe- 
riodic layered (planar) configurations by neglecting the construction details. Table 1 summarizes the major past work in studying wave profiles in alternating layered systems using specifically the plate impact test configuration. For almost all the experiments, stress (or velocity) response have shown a sloped rise portion followed by an oscillatory behavior in the steady pulse duration segment [4-9]. This behavior is conspicuously absent in homogeneous systems. As a matter of fact, the sloped rise portion and the oscillatory behavior about a mean value in the periodically layered systems are consistently exhibited in the systematic experimental work by Zhuang and coworkers [9, 10]. This confirms the statement by Oved et al. [6] that oscillations can be very significant and do not vanish in the shock regime, which is contrary to Barker's prediction [11]. Dandekar and Beaulieu [12] and Boteler et al. [13] reported results from plate impact tests on a woven fabric composite (GRP). The measured stress signal revealed also oscillatory peak stress behavior and a long rise time.

Among the modeling efforts, the mechanical behavior of composites has been extensively investigated using homogenization approaches. Since these approaches do not directly consider the interfaces, they are limited in examining the impact behavior, where the wave interactions can be very important. For example, consider the idea of replacing the dispersive, heterogeneous composites with an equivalent homogeneous dissipative continuum first proposed by Barker [11]. Barker was able to match some numerical results in the mean stress response with randomized thickness ratios. He showed using numerical simulations that the oscillations in the stress wave response disappear when the thickness ratios were randomized. However, as will be discussed later in this paper, his specific experiments [5] for validating his viscoelasticity-based theory cannot be generalized to other systems. Alternatively, micromechanics-based analysis of composites at the level of representative volume element (RVE), though has been widely used for modeling in-plane static behavior for lamina with complex architecture, has not been successfully applied for analyzing wave propagation in composites. For periodically layered systems, the latetime asymptotic solutions and the wave front solution for elastic and viscoelastic wave propagation normal to the layers subjected to unit step loading at the boundary were developed in 1970s [14-16]. El-Raheb [17, 18] developed approximate solutions for transient waves inside finite ordered or disordered bilaminates by deriving transfer matrices. The plate impact problem is an initial velocity boundary value problem, and the stress boundary condition on the surface of the layered medium keeps varying due to wave reflections at internal interfaces [19]. This is different from [14, 15] and El-Raheb's work where the stress boundary conditions were applied. Therefore, we first determined the stress
Table 1. Review of major past work done in studying wave profiles in alternating layered systems under plate impact

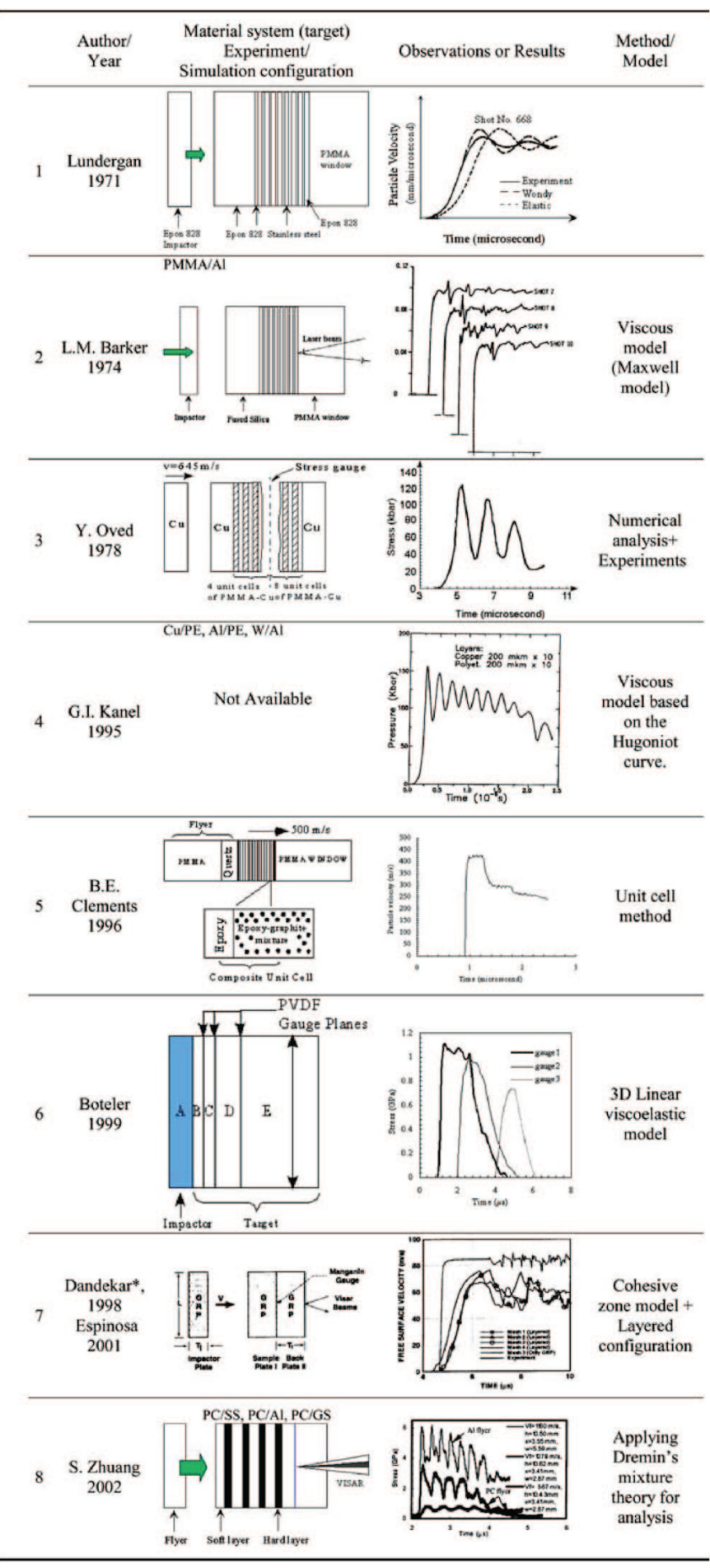

boundary condition and then developed a late time solution for both elastic and shock response based on both Floquet theory and mixture theory. The solution is validated by numerical and available experimental data. As clearly evidenced in Table 1 and other theoretical works, there is a lot of interest in understanding the dynamic behavior of composites under plate impact loading con- 
ditions. In most of the cases shown in Table 1, shock wave is generally generated since the impact velocity is sufficiently high. In these cases, various nonlinear effects may affect the observed behavior [20]. However, in nearly brittle material systems, our analytical solution shows that wave interactions still dominate and that the stress response can be predicted fairly well by invoking equation of state (EOS) of the materials in the periodic layered system.

The primary objective of this paper is to identify the main material heterogeneity factors that determine the high velocity impact response in periodically layered planar systems. The next objective is to examine the roles of these factors in determining the observed characteristics of the shock wave stress vs. time profile in terms of arrival time, rise time, peak stress and oscillatory pulse duration. For these purposes, we invoke the results of an analytical solution to the problem of plate impact test on periodically layered systems. In Section 2 , impedance mismatch, interface density and thickness ratio have been identified as three main heterogeneity factors that contribute to the observed stress wave profile. The relationship between engineering composites and layered systems in terms of these heterogeneity factors are examined. In Section 3, we proceed to analyze the effect of these heterogeneity factors on the structure of shock wave primarily based on the analytical solution developed previously [19]. We enumerate the important observations in the final summary section.

\section{Layered heterogeneous systems vs. composites}

\subsection{Controlling parameters under impact loading}

For a composite subjected to impact loadings, the impact response depends on the loading conditions and also the architecture of the composites. By architecture we imply the constructional details of the composites including fiber-matrix arrangement, stacking sequence and type and method of weaving. The loading condition generally involves the geometry of the impactor (e.g., bullet, plate, or ball), impact direction (normal or oblique) and impact velocity. The interaction of waves at an interface is relatively less complicated when the impact angle is normal and when the impact surface is planar. In plate impact tests, the impact velocity is a critical factor and it determines whether the material response falls into elastic, elastic-plastic or shock regime. When the local stress exceeds the hugoniot elastic limit (HEL), the material behavior falls into elastic-plastic regime. With further increase in stress levels, the material response enters the shock regime, where equation of state dominates the material response. In elastic-plastic and shock regime, material nonlinearity effects can also arise due to void nucleation and growth, microcracking and delamination.
Other than loading conditions, various characteristic parameters that determine the structure of a laminated composite will distinguish one composite from another dramatically. In this paper, we investigate both the elastic and the shock response of layered heterogeneous systems under plate impact loading conditions.

\subsection{Engineering composites vs. periodic layered systems}

Any propagating stress wave interacts in the form of reflections and transmissions whenever the wave encounters a heterogeneous interface. In engineering laminated composites, interfaces occur at all locations of material discontinuity and they arise at different length scales. Take the 2D woven composite laminates shown in Figure 1 for illustration [21-24]. The problem can be broadly classified as macro, meso and microlength scales. The macro applies to the entire laminated system in the range of a few millimeters and above. Mesoscale includes individual laminae, planar interlamellar interfaces at the length scales of a few hundred microns. Finally, the microscale refers to the length of a single fiber and includes fiber/ matrix interfaces inside the individual warp/fill tows in the range of a few micrometers. In general, the impact response due to wave interactions at interfaces can be very different from each other, since composites are made up of various fiber volume fractions, layup sequences, ply orientations or forms of fiber arrangements within the matrix (particulate, fibrous, textile), selected primarily to meet the various design needs. For a given composite system the rate of loading determines the characteristic length scale for the analysis.
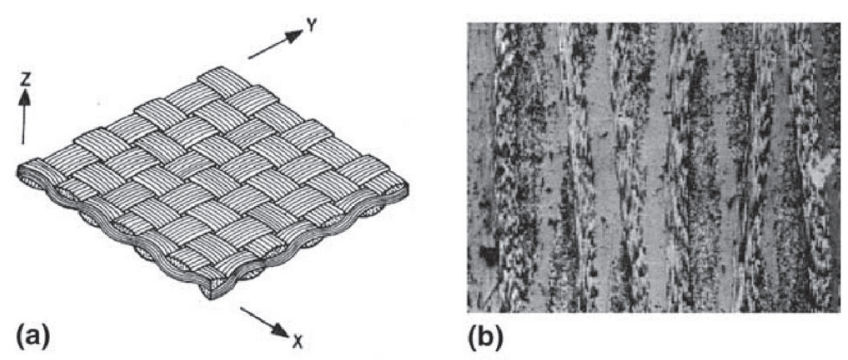

(b)

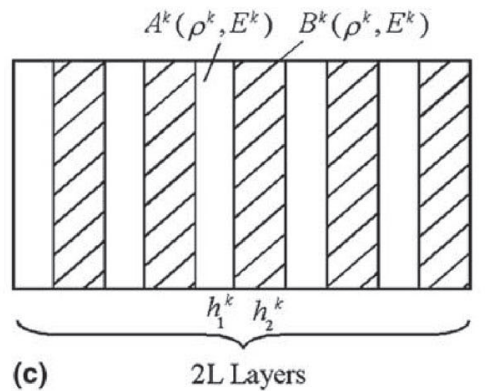

Figure 1. Microstructure of 2D woven structure: (a) fiber fabric, (b) lay-up sequence and (c) idealized alternating planar systems. 
The higher the loading rate, the smaller the length scale inside the composites that should be considered. When the rate of loading is almost quasi-static, macromechanics-based structural analysis is sufficient. In such a case, the whole plate which is a few centimeters thick can be considered homogenous and anisotropic with a characteristic interaction time of a few seconds. As the velocity of impact is increased, the wave interaction time decreases from a few seconds in the case of quasi-static loadings, to a few milli-seconds for low velocity impact loadings, e.g., drop weight test (impact velocity less than $20 \mathrm{~m} / \mathrm{s}$ ). In this case, macromechanics-based analysis at the level of lamina suffices. In plate impact tests, the velocity of the flyer plate can range from tens of meters per second to about 2000 $\mathrm{m} / \mathrm{s}(2 \mathrm{~km} / \mathrm{s})$. Generally the interaction time is in the range of a few tenths to a few microseconds. For these cases, consideration of mesoscale with the laminae as the appropriate length scale is sufficient. However, here one needs to invoke micromechanics-based analysis that explicitly considers the fabric/matrix interfaces. Homogenizing the lamina as an orthotropic medium is not sufficient. It is recognized that while investigation at the small length scales is required for understanding the micromechanisms, study at a larger length and time scales are preferred for efficient computational schemes. Therefore, micromechanics-based study at the lamina level is often conducted for composites made up of unidirectional plies. Herein, we are interested in two types of laminated composites: planar layered composites and 2D woven composites.

In this section, we examine engineering composites vis-a-vis layered material systems since the latter configurations have been predominantly used in plate impact tests. As shown in Section 1, extensive research has been done to study the wave propagation in periodic layered systems. In order to apply the theory and results from the layered systems to the engineering laminated composites, idealizations of laminated composites into periodically planar layered structure are necessary. The assumption in the model is that there exists a repeating unit cell (two alternating layers) with fixed thickness and material properties (shown in Figure 1(c)). Then the question left is to justify the idealization of 2D woven to a layered one ( Figure 1(b)). First, for the plain weave fabric shown in Figure 1(a), we restrict our analysis to the wave propagation in the thickness direction. So the interfaces between the warp tows and fill tows are ignored and also their material properties in the thickness direction are assumed to be the same. Second, the crimp angles of the yarns are usually very small, so that the effect of interface waviness is insignificant, justifying that we consider the fiber fabric as one layer of homogeneous orthotropic material. The corresponding constitutive relation for the fiber fabric should be determined using micromechan- ics-based model, which will be addressed elsewhere. Further, to simplify the structure of such composites, we can ignore the curved shape of the fiber fabric and the difference between the laminae; and consequently when the wave travels inside GRP, it travels through strictly periodic layers of pure matrix and fiber fabric. In this way, we assume that the model shown in Figure 1 (c) can be qualitatively applied to the $2 \mathrm{D}$ woven laminated GRP.

Description of engineering composites is quite complex and has led many researchers to work with periodically layered systems as an idealized representation of the composites. In the layered system as shown in Figure 1(c), the controlling parameters include the impact velocity $v_{0}$, Elastic moduli $E_{i}(i=1,2,3)$, Poisson's ratios $v_{i}(i=1,2,3)$, densities $p_{i}(i=1,2,3)$ and sound velocities $c_{i}(i=1,2,3)$, number of layers $2 L$ and thickness $h_{i}(i=1,2)$, respectively. Here, the subscripts $i=1,2,3$ represent the properties of materials $\mathrm{A}, \mathrm{B}$ and $\mathrm{C}$, respectively. $\mathrm{C}$ is the material for the flyer plate. A and $B$ represent the constituent layers of the target plate. From both the past work by current authors [25] and the analytical solution in a more recent paper [19], it can be easily seen that the origin of the observed structure of the stress waves can be attributed to material heterogeneity at the interfaces. The level of heterogeneity of a layered system depends mainly on impedance mismatch between $A$ and $B$, impedance mismatch between $\mathrm{A}$ and $\mathrm{C}$, characteristics of geometry arrangement (such as total target thickness, thickness ratio between two component materials, number of layers, stacking sequence). Here, we present the three factors that define material heterogeneity in layered systems: impedance mismatch, number of layers/interface density and the thickness ratio.

\subsection{Heterogeneity factors in periodic layered systems}

It is a common feature that in both composites and layered systems wave interactions play a very critical role. When wave encounters an interface, the strength of the reflected wave and the transmitted wave depends on the level of acoustic impedance mismatch. In layered systems, when multiple wave scattering takes place, other factors can also become important. We can identify three different factors that affect the wave interactions; these are termed as heterogeneity factors and can be categorized as

(1) Impedance mismatch

(2) Number of layers/interface density

(3) Thickness ratio

In the following section, we examine each of them carefully and discuss the effect of each of the factors on the response of material systems to high velocity impact loading. 


\subsubsection{Impedance mismatch}

Impedance mismatch factor $I$ can be defined as [26]

$$
I=1-\frac{4 Z_{1} Z_{2}}{\left(Z_{1}+Z_{2}\right)^{2}}
$$

where $Z_{1}$ and $Z_{2}$ are impedance of layers $A$ and $B$, respectively. Since the second term on the right-hand side represents the transmitted strength of waves after one unit cell, I represents the fraction of the incident stress that is not transmitted (i.e., reflected back) as the wave passes through a pair of $\mathrm{A} / \mathrm{B}$ and $\mathrm{B} / \mathrm{A}$ interfaces. $I=1$ represents infinite impedance mismatch with complete reflection, while $I=0$ leads to no mismatch with complete transmission. By rearranging the above equation, we can obtain the following relation

$$
I=\left(\frac{\rho_{1} c_{1}-\rho_{2} c_{2}}{\rho_{1} c_{1}+\rho_{2} c_{2}}\right)^{2}=r^{2}
$$

where $r$ is the reflection ratio at $\mathrm{A} / \mathrm{B}$ or $\mathrm{B} / \mathrm{A}$ interface.

Impedance ratio $\left(R=(\rho c)_{\text {hard }} /(\rho c)_{\text {soft' }}\right.$ i.e., the ratio of the impedance of the hard layer over that of the soft layer) is conventionally used to represent the level of impedance mismatch. From the definition, it is easily seen that $R$ ranges from 1 to infinity. When $R=1$, there is no impedance mismatch across the interface, and infinite $R$ represents infinite impedance mismatch. The relation between $I$ and $R$ is given by

$$
I=\left(\frac{R-1}{R+1}\right)^{2} \text {. }
$$

The relation given in Equation (3) is plotted in Figure 2. From this figure, it is seen that the impedance ratio $R$ has a one-to-one correspondence with impedance mismatch $I$. The higher the impedance ratio $R$, the higher is the value of $I$. However, quantitatively the relation is highly nonlinear. When the impedance ratio changes from 1 to 20, the corresponding impedance mismatch varies from 0 to 0.82 . But when the ratio continues to change from 20 to 40, the impedance mismatch varies very little by from 0.82 to 0.90 . It will be clearly shown that $I$ is a better measure than $R$, to represent mismatch in impedance.

It is useful to examine the effect of a given pair of materials on the magnitude $I$. When designing layered structures to resist impact, it is important to choose the right material combinations from a list of engineering materials. Table 2 lists the impedance mismatch values for 153 material combinations from 18 engineering materials, including organic materials and metals. The materials are listed such that the impedance ratio $R$ increases from left to right and from top to bottom. As a consequence, the impedance mismatch factors are distributed in the following order: $I$ increases from top to bottom in the same column and decreases from left to right in the same row. Since the combination that has maximum impedance mismatch in the table is

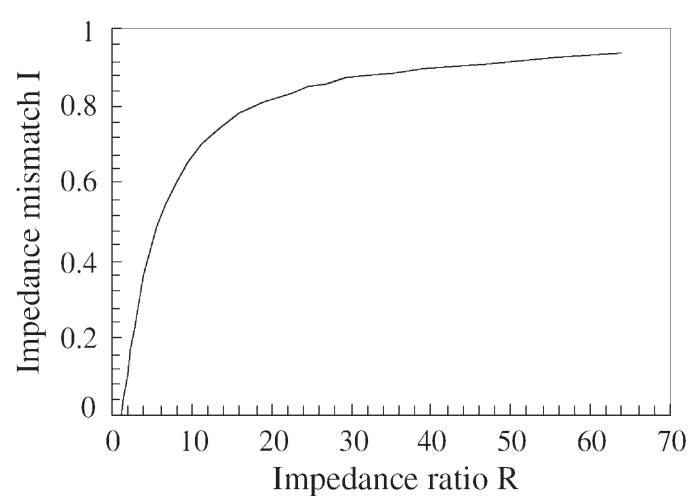

Figure 2. The relation between impedance mismatch $I$ and impedance ratio $R$.

Tungsten-Polyethylene with $I=0.933$ and the corresponding impedance ratio is about 57.6, we can expect that the impedance ratio for combinations of engineering materials will generally fall within the range of 1 and 60. We note that, Epoxy-Nylon, 2024Al-S2Glass, $\mathrm{Pb}$-Titanium, 304 Steel-Cu, Nickel-Steel and PlatinumMolybdenum have almost the same impedances with $I$ $\approx 0$. The scattering effect in these systems is expected to be very limited. In addition, it is clearly seen that the impedance mismatch values are randomly distributed between 0 and the maximum value 0.933 . Finally, it is very interesting to note that the magnitude of $I$ does not depend on whether the material is polymeric, metallic, intermetallic or ceramic. Impedance mismatch of materials that are "dissimilar" in general material categories can be higher than that belonging to the same category. For example, the impedance mismatch $I_{\text {Al-glass }}$ is about 0.005 , while $I_{\mathrm{Mg} \text {-Tungsten }}=0.671$, which is counter-intuitive.

\subsubsection{Number of layers/interface density}

Let us define the interface density as the number of interfaces/layers per unit width. If the thickness of the target is fixed, then the interface density is directly proportional to the number of layers and hence the two terms will be used interchangeably in our discussions.

Upon initial impact of flyer plate on the target plate, the incident wave with magnitude $\sigma_{0}$ is generated at the impact instant given by

$$
\sigma_{0}=\frac{\rho_{3} c_{3} \rho_{1} c_{1} v_{0}}{\rho_{3} c_{3}+\rho_{1} c_{1}} .
$$

The magnitude of the head wave after $2 L$ transmissions in a system, comprising alternating $L$ layers of $A$ and $L$ layers of $B$, is given by

$$
\sigma_{T 2 L}=(1-I)^{L} \sigma_{0}
$$

where $\sigma_{T 2 L}$ represents the strength of the head wave after propagating through $L$ unit cells. The second wave train that arrives at $x=2 L d$ is given by 


$$
\sigma_{T 2 L}^{(2)}=2 L \cdot I(1-I)^{L} \sigma_{0}+\sigma_{T 2 L} \cdot r(1+r),
$$

where $\sigma_{T 2 L}^{(2)}$ represents the strength of the second wave train and $r$ is defined in Equation (2). From these equations, it is clear that $I$ is a better measure than impedance ratio $R$ to provide a quantitative description of the effect of impedance mismatch on wave strength across an interface. The third wave train and later wave trains contain more number of waves and contain different propagation paths. As a result, the stress wave profiles are incremental stress steps with some specific time delays.

It is clearly seen that the strength of the head wave keeps decreasing when traveling through more unit cells (or when $L$ increases) as shown in Equation (5). This equation shows that for a system with fixed impedance mismatch, i.e., for a given material combination, more interfaces can dampen the strength of the head wave. The laminar acoustic dampeners and antimeteorite shields are made based on the amplitude decay of the head wave. However, more interfaces will not necessarily result in attenuation of the peak stress or the averaged stress amplitude, because of the arrival of more wave trains after the head wave. These secondary wave trains (wave trains that follow the head wave) can play an important role by increasing the stress amplitude when a location of interest is far from the impact plane ( $L$ is large). This is due to the fact that more interfaces lead to more number of wave interactions resulting in more number of waves in successive wave trains, as shown in Equation (6). In this equation, it can be seen that the impedance mismatch $I$ arises as a base, white the number of layers $L$ appears both as an exponent and as the coefficient. Thus, the quantitative effects of the two factors are quite different.

\subsubsection{Thickness ratio}

For a periodic bilaminated system, thickness ratio can be defined by $h_{1} / h_{2}$, and this quantity represents the volume fraction of the constituents in plate impact configuration. Here $h_{1}$ and $h_{2}$ are the thicknesses of materials $A$ and $B$ in a single unit cell. The effect of thickness ratio on the scattering process is to change the pattern of wave trains by altering the transit time and the total internal reflection sequence of the wave trains in each of the layers. Thus, for a given elapsed time, the number of waves traveling within the layers A or B is also a function of the thickness ratio. For the general case, we need to assume without any loss of generality, that one of the layers say $\mathrm{A}$ has a longer transit time $\left(t_{\mathrm{a}}\right)$ than that of layer $\mathrm{B}\left(t_{\mathrm{b}}\right)$. There is no change in the arrival of the first wave train and it is independent of the thickness ratio. The second wave train includes waves that go through one reflection in first layer A only. The content of subsequent wave trains depends on the specific value of the ratio of $t_{\mathrm{a}} / t_{\mathrm{b}}$. Take for instance, the third wave train; if the ratio $\left(t_{\mathrm{a}} / t_{\mathrm{b}}\right)>2$, then the third wave train comprises 


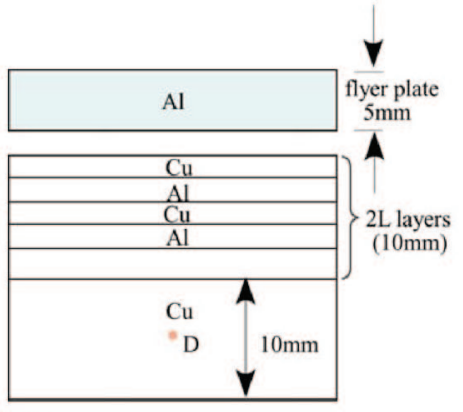

(a)
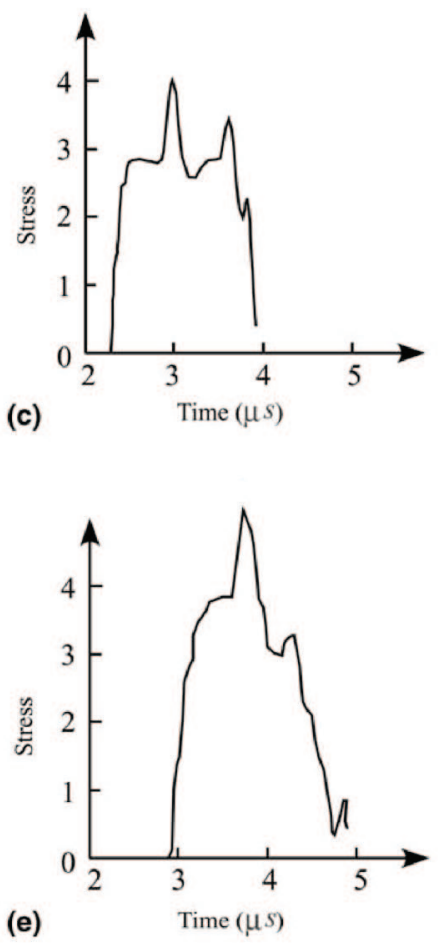

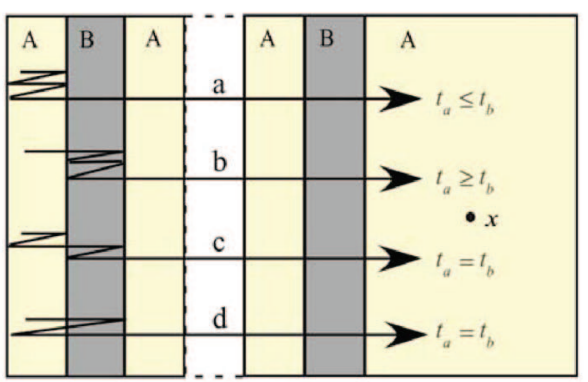

(b)

$\mathrm{L}+1$ layers $\mathrm{A}+\mathrm{L}$ layers $\mathrm{B}$

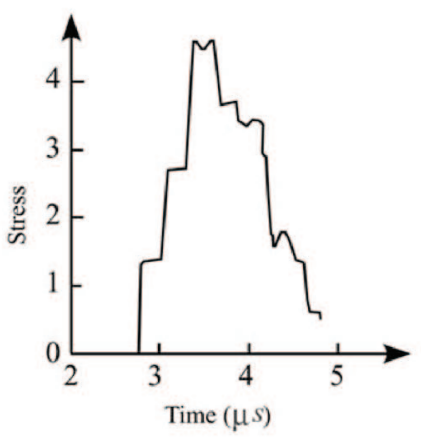

(d)

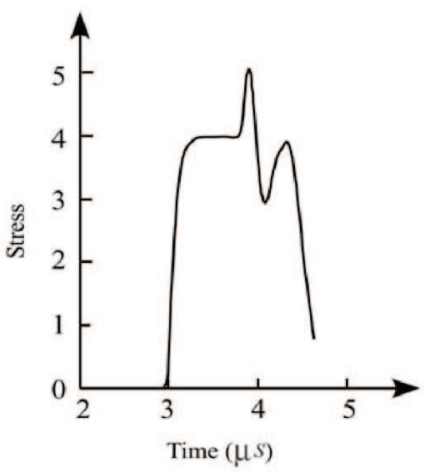

Figure 3. The effect of thickness ratio on the rise characteristics of Al-Cu structures: (a) configuration, (b) the third wave trains of different ratio, (c) $h_{1}=0.11 h_{2^{\prime}}$ (d) $h_{1}=1.5 h_{2^{\prime}}(\mathrm{e}) h_{1}=4.0 h_{2}$ and (f) $h_{1}=9.0 h_{2}$.

waves that go through two reflections in layer B. If, however, $\left(t_{\mathrm{a}} / t_{\mathrm{b}}\right)<2$, the third wave train comprises of waves that go through one reflection in layer A. Since the sequence of wave train is different, the observed slope and the oscillations are also different. It can be seen that the absolute value of $t_{\mathrm{a}} / t_{\mathrm{b}}$ indicates the wave train pattern more precisely than $h_{1} / h_{2}$. Figure 3 shows the numerical simulation of the effect of thickness ratio on the stress history downstream of four unit cells in an Al-Cu system (configuration is shown in Figure 3(a)). In this figure, when $h_{1} / h_{2}$ is moderate, (i.e./ the case of Figures 3(d) \& 3(e)), the stress increases in three steps to the peak value. The time intervals between the steps are obvious. On the contrary, when $h_{1} / h_{2}$ is very small or very large (i.e., the case of Figure 3(c) or Figure 3(f)), the stress wave profiles show smooth rise part. In this case, the shape of the stress profile in the rise part and duration part is very similar to the case of Barker's experi- ment (shown in Table 1, row 2), where the volume fraction of one constituent is very small with $\left(h_{2} / h_{1}\right)=20$.

If $h_{1} / h_{2}$ is very small, the second wave train, the third, the fourth, etc. arrive at a given location through reflections only in layer A. However, if $h_{1} / h_{2}$ is very large, then these wave trains go through reflections in layer $B$ only. For both cases, these wave trains are compressive. Since the time intervals between these wave trains are very small, the rise slope is sharp and the slope gradually decreases till a flat portion is reached. Evidently, we can see that the sharp rising slope is not due to small viscosity of the laminates as indicated in Barker's model, but due to the effect of low $h_{1} / h_{2}$ ratio [5].

Barker suggested that when the thickness of the layers were randomized, the stress oscillations are largely removed. This phenomenon can also be explained by the disturbance of the wave trains. When the structure is completely randomized, the same wave trains include 
both tensile and compressive components. Besides, the magnitude and the arrival times of these waves are totally randomized, leading to the randomization of the wave pattern. As a consequence, the net effect is that cumulative stress remains constant and thus stress oscillations are no longer periodic or significant. For engineering composites, the thickness ratio is not a constant due to factors like stacking sequence, so the oscillations tend to become arbitrary.

\subsection{Heterogeneity factors in composites}

We have identified three different heterogeneity factors that characterize layered systems. Though the real composites are also characterized by impedance mismatch, interface density and volume fraction, we have not established quantitative relations to link these factors to engineering composites. This is a formidable task compounded by the fact that there is a lack of experimental data of plate impact tests on engineering composites. Though we could not establish an exact quantitative relationship, it is clear that the three factors will qualitatively affect the impact response of engineering composites in a similar fashion.

\section{Effect of material heterogeneity on the structure of the stress wave profiles}

Though the stress (or particle velocity) response has been very well understood for homogeneous materials, the same cannot be said for heterogeneous systems. A schematic of the shock wave profiles of homogeneous and layered systems are shown in Figure 4. Figure 4(a) shows a steep rise (AC) for elastically loaded material while $\mathrm{ABD}$ indicates the response for an elastic-plastic (AB-BD) material. BD changes slope for a rate-dependent inelastic material. At D (or C), the material is completely stressed to the peak stress value corresponding to the given impact velocity. E indicates the arrival of a tensile release (unloading) wave either from the rear surface of the flyer or target plate. Thus, EFGH indicates the pull-back signal. Figure 4(b) shows the schematic of the typical shock response of a layered composite system. $A_{1} R_{1}$ shows a sloped rise portion in the profile. Stress oscillations in the pulse duration segment $\left(R_{1} T_{1} R_{2} T_{2} E_{1}\right)$. As can be seen in Table 1 , for different layered systems, the rise time can be small or finite; the oscillations can be substantial or negligible. It should be noted that these variations have neither been explained nor predicted by homogenization-based theories or models. The reason is quite simple, wave scattering at interfaces has not been explicitly accounted for in those theories or models. In the following section, we point out that the observed stress wave profiles can be explained by analyzing wave scattering.
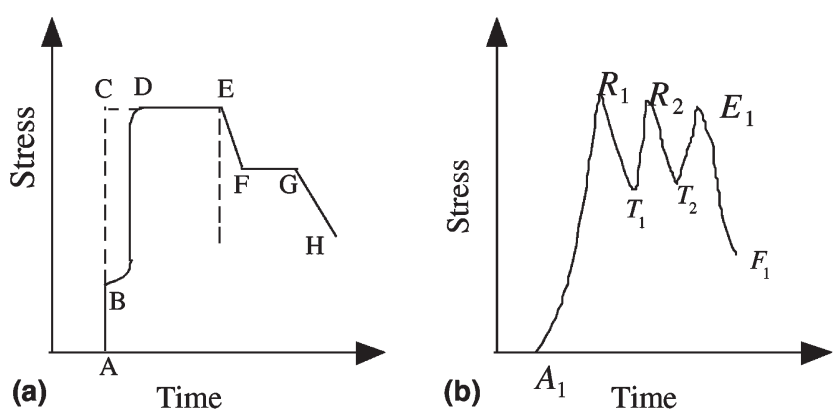

Figure 4. Schematic wave profiles of homogeneous metals (a) and layered composites (b) for a finite thickness flyer plate.

\subsection{Stress wave profiles as results of wave interactions at interfaces}

It was shown [25] that the stress wave profile at any given propagation distance inside the layered target is directly due to sequential arrivals of wave trains. Though this general idea is valid at all locations, tracing the number of waves with specific propagation paths can be very cumbersome when the system comprises a large number of layers. In such cases, the analytical solution provided in an recent paper [19] should be used to predict the stress response. In this section we briefly present the solution for the sake of completeness. Though detailed derivations are provided elsewhere [19], the necessary equations for solving the problem are outlined here. This solution solves the problem in which a semi-infinite body $\Omega_{1}(-\infty<y, z<\infty, 0 \leq x<\infty)$ impacts another semiinfinite body $\Omega_{2}(-\infty<y, z<\infty,-\infty<x<0)$ at $x=0$ plane with velocity $v_{0}$ (as shown in Figure 5(a)). This problem is identical to the problem in Figure 5(b) if the thickness of the flyer plate $\left(d_{\mathrm{f}}\right)$ and the target plate $\left(d_{\mathrm{t}}\right)$ are much smaller compared to the lateral dimensions (radius of the plates). However, both the thickness $d_{\mathrm{f}}$ and $d_{\mathrm{t}}$ are large enough not to permit the wave reflections from the free surfaces to interfere with the planar longitudinal waves. In this solution, the wave trains at the impact plane are monitored and they determine the loading conditions of the target plate. Figure 5(a) shows the schematics of the wave traveling within the target body $\Omega_{1}$. As the incident wave travels in material $\mathrm{A}$ and reaches the interface $A-B$, part of it is reflected back and the rest of it is transmitted (shown in dotted line only for wave train a). This reflected wave arrives back at the impact plane after a time $t_{\sigma 1}=2 t_{\mathrm{a}}=2 h_{1} / c_{1}$. Stress at the impact plane is altered by this new wave arrival given by

$$
\Delta \sigma_{1}=r_{\mathrm{A}-\mathrm{B}}\left(1+r_{\mathrm{A}-\mathrm{C}}\right) \sigma_{0}
$$

where $r_{\mathrm{A}-\mathrm{B}}$ denotes the reflection ratio in layer $\mathrm{A}$ at interface $\mathrm{A}-\mathrm{B}, r_{\mathrm{A}-\mathrm{C}}$ represents the reflection ratio in layer $\mathrm{A}$ at interface $\mathrm{A}-\mathrm{C}, \mathrm{C}$ is the flyer plate. So, this wave train is the second wave train that propagates in the layered system. The cumulative stress level at $x=0$ at $t_{\sigma 1}=2 t_{\mathrm{a}}=2 h_{1} / c_{1}$ is $\sigma_{1}=\sigma_{0}+\Delta \sigma_{1}$. The third, fourth and 

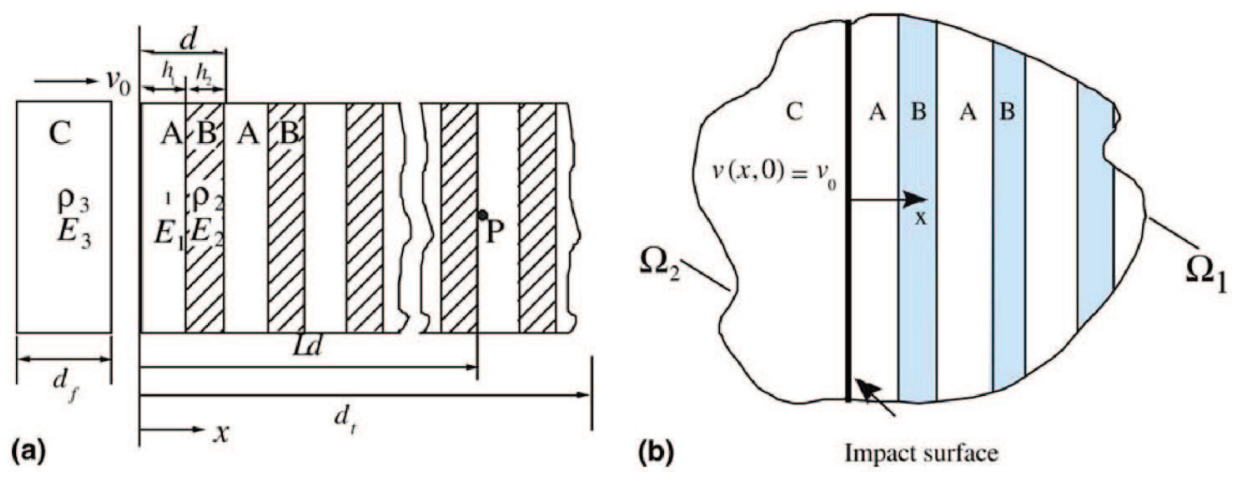

Figure 5. Schematic of the configuration for impact problem: (a) general plate impact problem of layered systems and (b) plate impact problem of two half spaces.

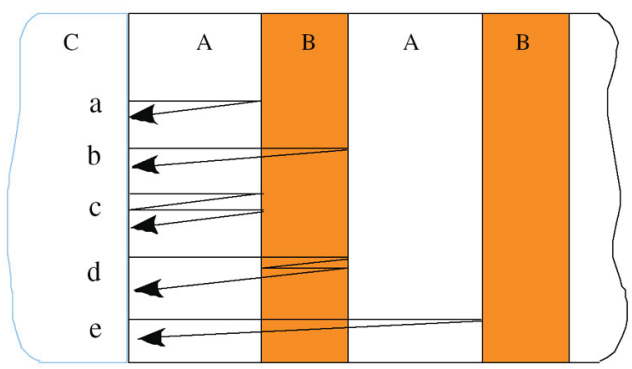

(a)

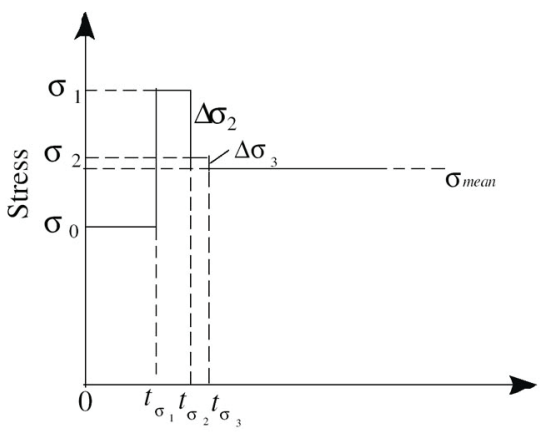

(b)

Time

Figure 6. Multi-step loading method: (a) the wave trains that reach the impact plane from inside of the target due to reflections and (b) the equivalent loading history of the target plate.

additional wave trains lead to incremental stresses $\Delta \sigma_{2}$, $\Delta \sigma_{3}, \ldots$ as shown in Figure 6(b). Thus, the stress boundary condition at $x=0$ comprises impact stress $\sigma_{0}$ (called the head wave), followed by the second wave train $\Delta \sigma_{1}$ after time $t=2 t_{\mathrm{a}^{\prime}}$, then the third wave train $\Delta \sigma_{2}$ at $t=2 t_{\mathrm{a}}$ $+2 t_{\mathrm{b}}\left(\right.$ if $\left.t_{\mathrm{a}}>t_{\mathrm{b}}\right)$ or at $t=4 t_{\mathrm{a}}\left(\right.$ if $\left.t_{\mathrm{a}} \leq t_{\mathrm{b}}\right)$ and so on. The exact solution should consider all these stress increments with specific time delays as the boundary condition at $x=0$. Since the formulation is linear, the late-time solution to plate impact problem can be obtained by the method of superposition of unit step loadings with steps corresponding to incremental stress and specific time delays.

It can be shown that a steady stress state or mean stress $\sigma_{\text {mean }}$ exists at the impact plane as a result of wave reflections and this was verified through a comparison of the analytical solution with the numerical solution. $\sigma_{\text {mean }}$ can be obtained by summing up the stress increments, or simply by invoking mixture theory. The mean stress can be obtained as

$$
\sigma_{\text {mean }}=\frac{\rho_{3} c_{3} \tilde{\rho}_{0} c_{0} v_{0}}{\rho_{3} c_{3}+\tilde{\rho}_{0} c_{0}},
$$

where $\tilde{\rho}_{0}$ is the effective density of the target plate with $\tilde{\rho}_{0}=\left(\rho_{1} h_{1}+\rho_{2} h_{2}\right) /\left(h_{1}+h_{2}\right) \cdot c_{0}$ is the effective wave velocity of the layered system and $c_{0}$ is given by

$$
c_{0}=\frac{h_{1}+h_{2}}{\left[\left(\frac{h_{1}}{c_{1}}\right)^{2}+\left(\frac{h_{2}}{c_{2}}\right)^{2}+\left(\frac{\rho_{1} c_{1}}{\rho_{2} c_{2}}+\frac{\rho_{2} c_{2}}{\rho_{1} c_{1}}\right) \frac{h_{1} h_{2}}{c_{1} c_{2}}\right]^{1 / 2}} .
$$

Now, we propose a solution comprising $n$ steps of stress increments due to the first $n$ wave trains. In order to make the final steady-state reach $\sigma_{\text {mean' }}$ we set $\Delta \sigma_{n-1}=$ $\sigma_{\text {mean }}-\sigma_{n-2}$. So for the four-step method (when $n=4$ ), the whole loading history at the impact plane is given by Figure 6(b), and the stress history can be written as

$$
\sigma(x, t)= \begin{cases}\sigma_{0}(x, t), & \frac{x}{c_{0}} \leqslant t<t_{\sigma_{1}}+\frac{x}{c_{0}}, \\ \sigma_{0}(x, t)+\Delta \sigma_{1}(x, t), & t_{\sigma_{1}}+\frac{x}{c_{0}} \leqslant t \leqslant t_{\sigma_{2}}+\frac{x}{c_{0}} \\ \sigma_{0}(x, t)+\Delta \sigma_{1}(x, t)+\Delta \sigma_{2}(x, t), & t_{\sigma_{2}}+\frac{x}{c_{0}}<t<t_{\sigma_{3}}+\frac{x}{c_{0}} \\ \sigma_{0}(x, t)+\Delta \sigma_{1}(x, t)+\Delta \sigma_{2}(x, t)+\Delta \sigma_{3}(x, t), & t_{\sigma_{3}}+\frac{x}{c_{0}}<t,\end{cases}
$$


where

$$
\begin{aligned}
& \sigma_{0}(x, t)=\sigma_{0}\left[\frac{1}{3}+\int_{0}^{B} A i(-s) \mathrm{d} s\right] \\
& \Delta \sigma_{j}(x, t)=\Delta \sigma_{j}\left[\frac{1}{3}+\int_{0}^{B} A i(-s) \mathrm{d} s\right] \\
& j=1,2,3,4 \\
& B=\left(t-\frac{x}{c_{0}}\right)\left(\frac{2}{h^{\prime \prime \prime}(0) t}\right)^{1 / 3}, \\
& h^{\prime \prime \prime}(0)=\frac{\left(c_{0}\right)^{2}}{d^{2}}\left\{\left(\frac{h_{1}}{c_{1}}\right)^{2}\left(\frac{h_{2}}{c_{2}}\right)^{2}\left[\frac{1}{4}\left(\frac{\rho_{1} c_{1}}{\rho_{2} c_{2}}+\frac{\rho_{2} c_{2}}{\rho_{1} c_{1}}\right)^{2}-1\right]\right\} .
\end{aligned}
$$

Equations (13) and (14), thus, summarize the solution to the plate impact problem when the target behavior is in the elastic regime.

\subsubsection{Extension to shock response by invoking EOS}

Shock response of materials is a highly nonlinear process and is extremely difficult if not impossible to obtain purely analytical solutions. In [19], an approximate solution based on the previously developed elastic solution was obtained for shock loading cases by invoking hydrodynamic treatment ignoring shear stresses. It is important to note that we are dealing with shock response and not the elastic-plastic response with the two-wave structure. We extend the elastic analysis to shock response by incorporating the nonlinear effects through computing shock velocities of the wave trains and superimposing them.

For laminated systems under shock loading, we need to use the equation of state, in which shock velocity $U_{s i^{\prime}}$ density $p_{i}^{\prime}$ and thickness $h_{i}^{\prime}(i=1,2)$ are related to the particle velocity. Then the effective velocity of the laminates for shock loading condition $\tilde{U}_{\mathrm{s}}$ can be obtained. Based on these new values, we obtain the mean stress level $\sigma_{\text {mean }}^{\prime}$ for layered systems under shock loading. In addition, when using multi-step loading method, modifications are needed when the secondary wave trains can overtake the wave front at a sufficiently large propagation distance $x$. The details are provided in Appendix A.

The above method of using EOS is used to simulate plate impact tests that corresponds to experimental data by Zhuang $[9,10]$. The set-up of his work is schematically shown in row 8 of Table 1 . The stress response was measured at an intermediate center line position using manganin gauges. Figure 7(a) shows the comparison of the experimental result with the analytical solution. In this experiment (Experiment No. 1), a flyer made of polycarbonate $(\mathrm{PC})$ impacts on a target made of alternating PC and glass with velocity of $1079 \mathrm{~m} / \mathrm{s}$. The test parameters are given in Table 3 . It can be seen that the mean stress obtained from analytical solution agrees well with the experimental result. The matching of experimental and analytical results is quite good, in terms of arrival time, peak stress, frequency of the oscillations and mean stress. Yet another experimental comparison (Experiment No. 2) is shown in Figure 7(b). This time a
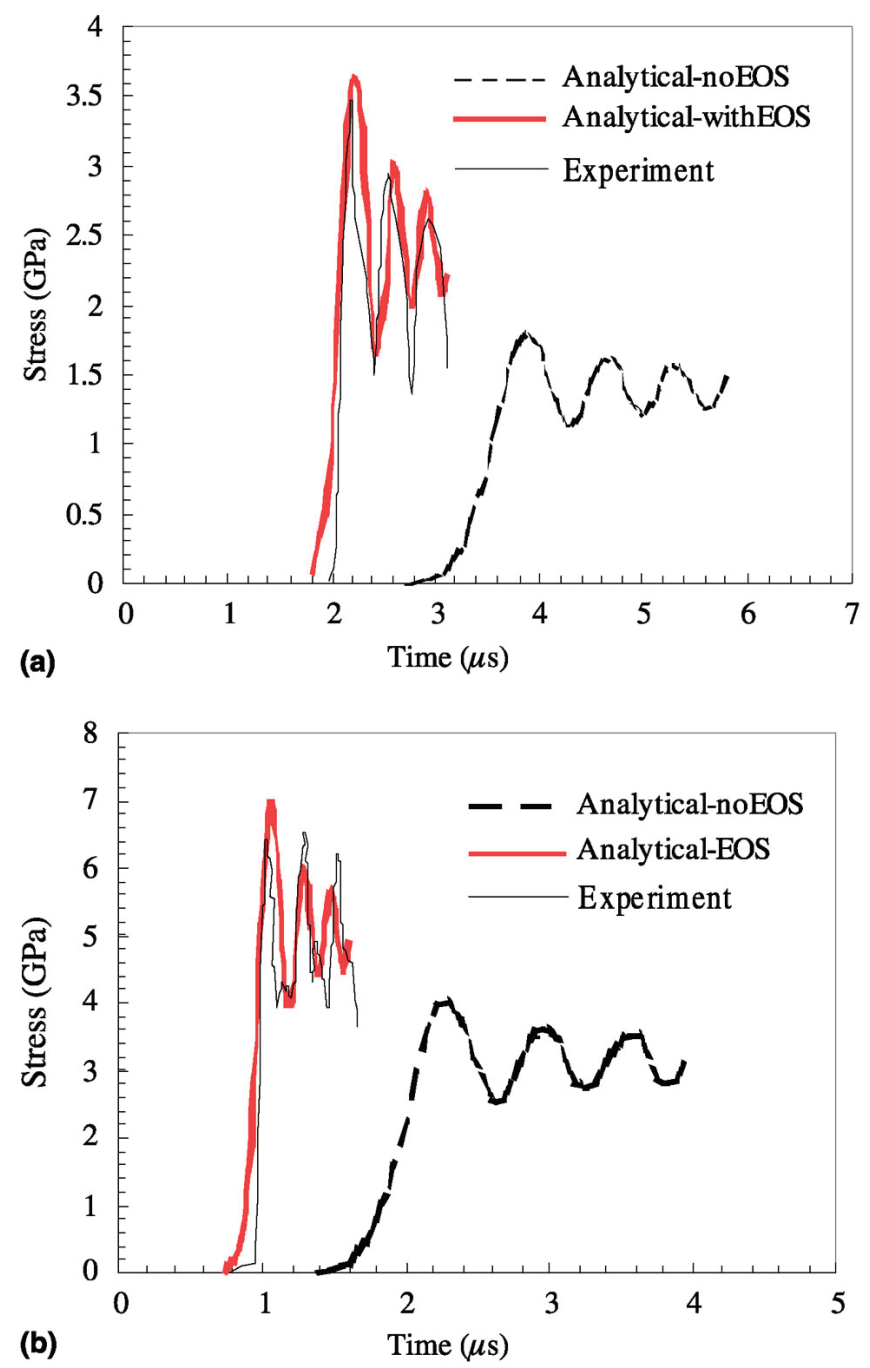

Figure 7. Comparisons of the experimental data and the analytical solutions in layered PC/GS: (a) Experiment 1 and (b) Experiment 2 [10].

Table 3. Configurations of Experiments 1 and 2 [10]

\begin{tabular}{llllllll}
\hline Experiments & A & B & C & Impact velocity $(\mathrm{m} / \mathrm{s})$ & $h_{1}(\mathrm{~mm})$ & $h_{2}(\mathrm{~mm})$ & Gauge location $x$ \\
\hline 1 & PC & Glass & PC & 1079 & 0.37 & 0.20 & 6.44 \\
2 & PC & Glass & A1 & 1160 & 0.37 & 0.20 & 3.55 \\
\hline
\end{tabular}



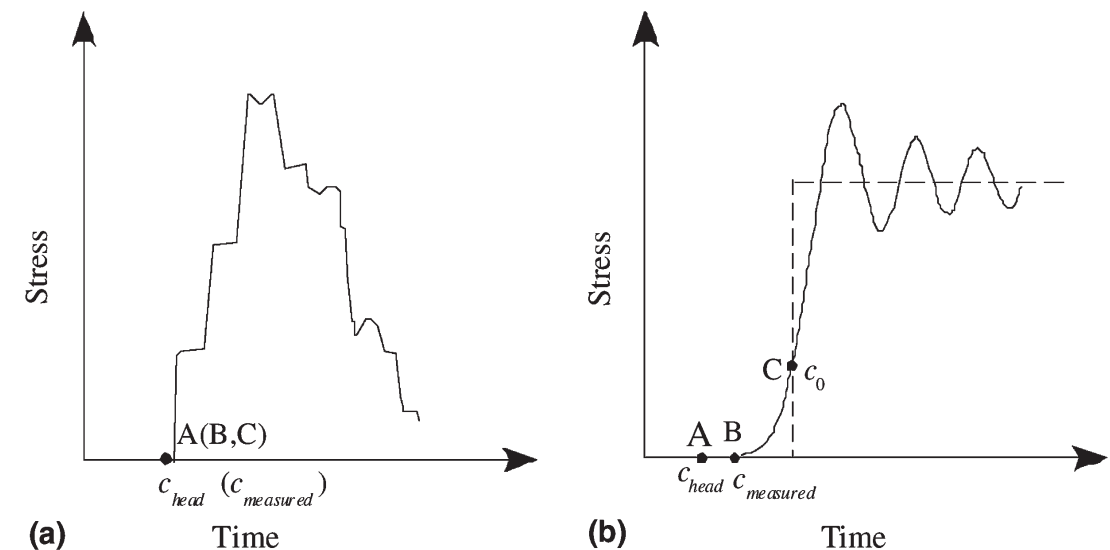

Figure 8. Schematics of the relationship between $c_{\text {head }} c_{\text {measured }}$ and $c_{0}$ : (a) $x \leq x^{*}$ and (b) $x>x^{*}$.

metal (Aluminum) with $5.59 \mathrm{~mm}$ in thickness, impacts on PC/GS with $1160 \mathrm{~m} / \mathrm{s}$. Again the overall agreement is good. In both analytical solutions, the EOS of components are the corresponding constitutive relations. We assumed that the materials do not exhibit plasticity and also are damage and defects-free.

The good agreement between the experimental data and the theoretical solution (Equations (10), (13), \& (14)) clearly shows that the proposed method can be used for plate impact tests in both elastic and shock loaded conditions. Though numerical simulation using finite element-based methods can also reproduce the experimental results, analytical solution has the distinct advantage of delineating the effects of various material, geometric and loading parameters on the wave structure and hence the material response. In the following section, we carefully examine the effect of each of the heterogeneity factors on the key characteristics of the wave profile.

\subsection{Rise characteristics}

\subsubsection{Arrival time}

Recent plate impact experiments by Zhuang [9] and numerical solutions have shown an "anomalous" phenomenon that the measured shock velocities of the PCGlass and PC-SS (stainless steel) systems were even lower than that of either PC or glass or SS. This indicates that the arrival time of the wave at a location in the laminate can be later than in any of the materials, if it were used alone. Here

$$
c_{\text {measured }}<c_{1} \text { and } c_{\text {measured }}<c_{2}
$$

For a layered system, $c_{\text {measured }}, c_{1}$ and $c_{2}$ are the wave velocities of the effective layered system (measured value), material A and material B, respectively.

Wave speeds of laminates both in shock and elastic regimes are experimentally computed by recording the arrival time and distance of propagation from the impact plane. Once we know the time and distance of travel, the speed can be calculated. In the following section, the phenomenon of wave propagation is used to explain the anomaly.

3.2.1.1. Elastic waves. If $c_{\text {head }}$ is the velocity of the head wave for a single unit cell, then

$$
c_{\text {head }}=\frac{h_{1}+h_{2}}{\frac{h_{1}}{c_{1}}+\frac{h_{2}}{c_{2}}} .
$$

If we assume that $c_{1}>c_{2}$, we can be see that if volume fraction of material A (with high velocity) increases (higher $h_{1} / h_{2}$ ), then the $c_{\text {head }}$ will increase. The equation also demonstrates that $c_{\text {head }}$ will always lie in between $c_{1}$ and $c_{2}$. The observation simply means that the magnitude of the head wave is so small, the first arrived (measurable) wave corresponds to some reflected/transmitted wave train. As a matter of fact, it can be shown [25] that for a target system with big impedance mismatch, the wave front quickly dies out. For example, for systems with $I=0.8$, the strength of the head wave reduces to $\sigma_{2 L T}=4.0 * 10^{-9} \sigma_{0}$ after 12 unit cells. With such low stress level, obviously the measuring equipment cannot properly capture the arrival of the head wave. One can always determine a characteristic location $x^{*}$ in the sample such that the stress strength will be lower than the sensitivity $\varepsilon$ of the measuring device, i.e., $\left|\sigma_{2 L T}\right|<$ $\varepsilon$. Thus, if the thickness of the sample corresponding to measurement location is greater than $x^{*}$, then the measured velocity $c_{\text {measured }}$ will be lower than $c_{\text {head }}$. In other words, we are not truly measuring the head wave arrival time in such a case. Consequently, the statement that wave velocity can be lower than either of the material velocities can be confusing if the underlying physics is not provided. When the measuring location is less than $x^{*}$, this implies that the head wave is still the measured wave and this case is schematically shown in Figure 8(a).

Here, we examine the situation when $x \gg x^{*}$, wherein the head wave has died down and stress signal is due to subsequent wave trains. At these locations, the late time 


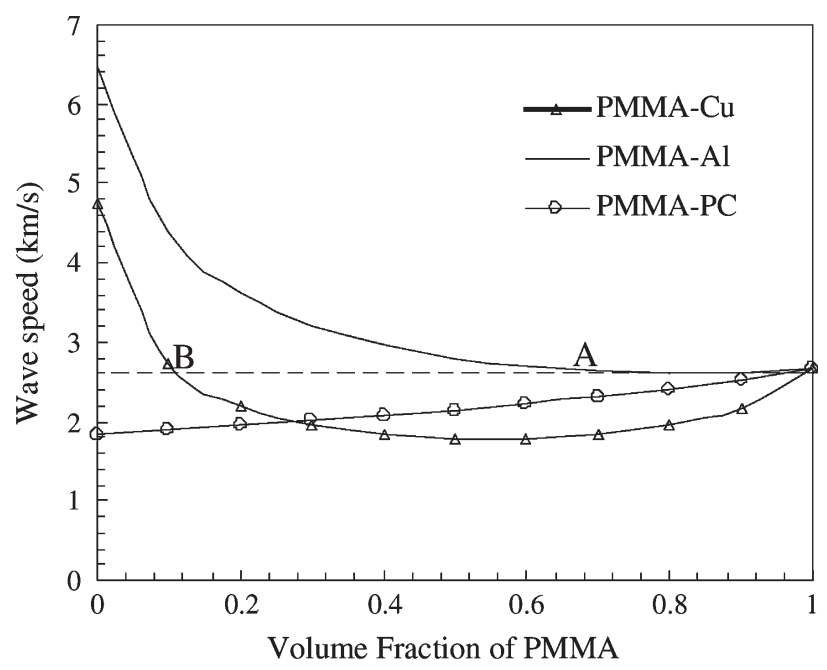

Figure 9. The effective velocity $c_{0}$ of different systems.

solution given by Equations (9), (10), (13), and (14) are valid. $c_{0}$ as given by Equation (9) represents the wave velocity of the main disturbance in the far field [16]. By examining Equations (11) and (13), it can be seen that the stress level corresponds to $t=x / c_{0}$, where $B=0$ and $\sigma=(1 / 3) \sigma_{\text {mean }}$. See Figure 8(b) for a schematic illustration of the phenomenon. The measured velocity $c_{\text {measured }}$ is very close to $c_{0}$ since typically the time difference between $\sigma=0$ to $\sigma=(1 / 3) \sigma_{\text {mean }}$ is very small. Hence, the measured velocity $c_{\text {measured }}$ corresponds to $c_{0}$ in elastic cases. And by examining the variation of $c_{0}$, we can explain the variations in the measured wave velocities $c_{\text {measured }}$. Figure 9 shows the variation of $c_{0}$ as a function of volume fraction of PMMA in various PMMA-based composites (PMMA-polycarbonate, PMMA-Al and PMMA-Cu). The longitudinal velocity of PC, PMMA, $\mathrm{Al}$ and $\mathrm{Cu}$ are $1.85,2.66,6.473$, and $4.74 \mathrm{~mm} / \mu \mathrm{s}$, respectively. Their impedance mismatches are $I=0.03,0.507$, and 0.743 for PMMA-PC, PMMA-Al, and PMMA-Cu, respectively. The left extremes of each of the curves represent the velocities corresponding to $\mathrm{PC}, \mathrm{Cu}$, and $\mathrm{Al}$ while the right end represents the velocity of PMMA. When the impedance mismatch is low (as in the case of PMMA-PC), the velocity $c_{0}$ stays in between the two extreme values. However, as the impedance mismatch increases, there is an inverted bell-shaped curve with the lowest $c_{0}$ occurring at some intermediate values of matrix volume fraction. This fact has led investigators to the assumption that wave velocities decreases in some laminated system below that of its constituents. The effect of impedance mismatch on $c_{0}$ can be further analyzed by examining Equation (9). For the case of no impedance mismatch (homogeneous with $\rho_{1} c_{1}=\rho_{2} c_{2}$ ), $c_{0}=$ $c_{\text {head }}$. When $\rho_{1} c_{1} \neq \rho_{2} c_{2},\left(\rho_{1} c_{1} / \rho_{2} c_{2}\right)+\left(\rho_{2} c_{2} / \rho_{1} c_{1}\right)>2$ and $c_{0}<$ $c_{\text {head }}$. When $\left(\rho_{1} c_{1} / \rho_{2} c_{2}\right)+\left(\rho_{2} c_{2} / \rho_{1} c_{1}\right) \gg 2$ (impedance mismatch is very high), $c_{0} \ll c_{\text {head }}$. It can be seen that there will be a critical value of volume fraction when the min- imum $c_{0}$ occurs. These explanations are accurate for elastic waves.

3.2.1.2. Shock waves. There are two additional factors that complicate the determination of arrival time when the waves are in the shock wave regime.

1. The velocity of the shock wave decreases with decrease in the magnitude of the stress. Since the wave interacts at every interface, the strength of the wave (stress levels) decreases with the number of unit cells (the propagating distance $x$ ). Since the velocity of the shock wave continues to decrease in laminated systems, there is a delay in the arrival time.

2. There is a counter-acting effect to the above phenomenon. The material behind the head shock wave is in a state of high level of compression. Shock waves travel faster in a compressed medium. So the wave trains behind the head wave (as a result of interfacial wave interaction) travel faster than the head wave and can catch up with the head wave. Upon catching, the combined waves travel faster than the original head wave velocity. The arrival time obviously depends on whether the subsequent waves have the time (and hence $x$ ) to catch up with the original head wave. Catching-up process depends on the impact velocity, interface density, impedance mismatch and the location of measurement, so the arrival time also depends on these factors in the shock wave regime.

\subsubsection{Rise time}

We define the rise time as the time difference between the time of arrival of the head wave (measurable) and the time when the first peak stress value is reached. Previous experiments have shown that the rise time under elastic response of metals is about 20-30 ns [27]. Finite rise time under elastic-plastic response of metals are usually attribute to dislocation related mechanisms. In layered heterogeneous materials, much longer rise time has been reported that is much more than any of the constituents acting alone. This is true even for elastically loaded cases on layered systems, including metal-based composite system. Figure 10 shows the complete stress vs. time profile for three different layered systems that were previously studied. As noted earlier, these systems have a wide range of impedance mismatch values. In Figure 10(a), the response is plotted when the layered system is impacted by PMMA, while Figure 10(b) shows the plot when the impactor is aluminum. The thickness ratio is fixed at $h_{1} / h_{2}=(0.26$ $\mathrm{mm} / 0.36 \mathrm{~mm})$, and the response is plotted at a location $x=10 \mathrm{~mm}$. All the cases exhibit a finite rise time, while a single target material would have (virtually) no rise time. Rise time increases with increase in impedance mismatch. Comparing Figure 10(a) and 10(b), it can be seen that the rise time is unaffected by the choice of the material of the flyer plate. This is obvious since the flyer 

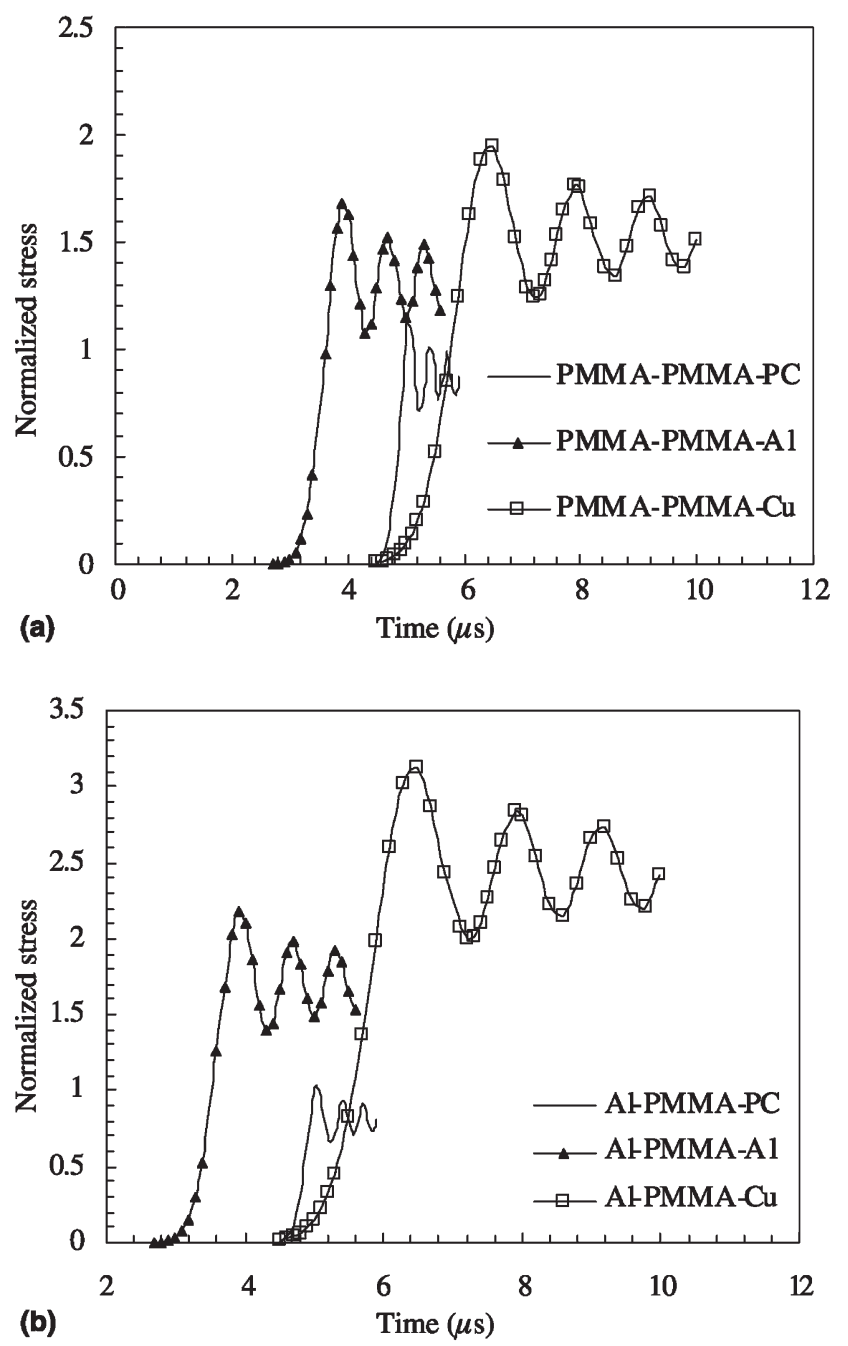

Figure 10. The analytical solutions to the normalized stress history (with respect to $\sigma_{0}$ ) in PMMA-PC, PMMA-Al and PMMA-Cu: (a) Flyer plate is PMMA and (b) Flyer plate is Al.

plate material changes only the magnitude of the stress at the impact plane but not the wave pattern. Averaged rise slope can be defined by the average of the slope of $\sigma-t$ curve $(\mathrm{d} \sigma / \mathrm{d} t)$ up to the peak, which can be approximated by $\Delta \sigma_{\text {peak }} / \Delta t_{\text {rise }}$. It should be noted that while flyer plate material affects the rise slope, rise time stays unchanged. This is obvious since different levels of peak stresses are obtained with different impactors but the rise time, which is governed by the target plate, remains unaltered.

We can further obtain additional insights into rise time by examining integral limit parameter $B$ in the equation,

$$
\sigma(L d, t)=\sigma_{\text {mean }}\left[\frac{1}{3}+\int_{0}^{B} A i(-s) \mathrm{d} s\right] .
$$

Here, $B$ is still given by Equation (13). We can identify the rise time as the time interval between the time the minimal stress is measured to that when the peak stress is reached. A simple numerical analysis shows that the integral limit $B \approx-3.3$ corresponds to $-(1 / 3)$ in the in-

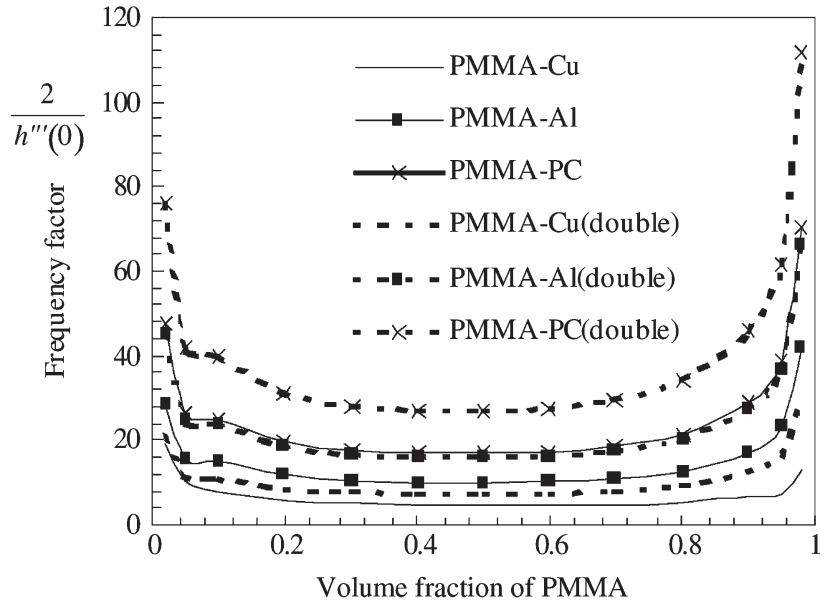

Figure 11. The frequency factors of different layered systems.

tegral (Equation (17)) such that the stress level just becomes measurable. Further when $B \approx 2.3$, the peak stress is reached. Thus, the time difference between $B \approx-3.3$ $\left(t_{\text {initial }}\right)$ and $B \approx 2.3\left(t_{\text {peak }}\right)$ yields the rise time $\left(t_{\text {peak }}-t_{\text {ini- }}\right.$ tial). Since $B$ is distance- $x$ and time- $t$ dependent, it is clear that the rise time is $x$-dependent. As $x$ increases, the rise time also increases. For a fixed location $x$, the rise time then depends on the factor $2 / h^{\text {tprime }}(0)$ in Equation (13). The factor $2 / h^{\prime \prime \prime}(0)$ determines how fast $B$ changes, and is termed in this paper as the frequency factor since it also governs the frequency of oscillations. The higher the factor, the shorter the rise time and the higher the frequency of oscillations (to be discussed later). Figure 11 plots the effects of impedance mismatch, interface density and thickness ratio due to Equation (14) in a few systems (volume fraction of PMMA is used as the variable). It can be seen in the figure that the effect of thickness ratio is not significant on the frequency factor whenever the volume fraction of either material is not negligibly low. In other words, two extreme systems with almost all PMMA or nearly no PMMA will both result in low rise time. Thus, a quasi-homogeneous material behavior is expected. The more important factor that affects the frequency factor is impedance mismatch (between materials $\mathrm{A}$ and $\mathrm{B}$ ); low impedance mismatch, such as PMMA-PC, will result in high frequency factor and short rise time, as shown in Figure 11. With the increase of the impedance mismatch, the frequency factor becomes lower. Also, the effect of interface density can be as critical as impedance mismatch for the rise time; with the increase of interface density, the rise time becomes shorter.

When loading leads to shock rather than the elastic waves, the analysis becomes more complicated since stress levels and wave velocities depend nonlinearly on the impact velocity. However, it can be noted that the rise time decreases with increase in impact velocity. But the trend that rise time increases with increase in impedance mismatch is still valid. 


\subsubsection{First peak}

In general, the first peak of the stress wave profiles (such as point $R_{1}$ in Figure 4) is usually the highest peak in the pulse duration segment. Since material damage may depend on the instantaneous stress levels, if peak stress is substantially higher than the average stress, it is a cause for concern. It is shown that stress at a given point in the domain is the net effect of all the incremental stress reaching that point from various sources of interfacial wave interactions, so the peak stress is reached when substantial levels of tensile stress wave arrive at that point.

As mentioned before, the late time solution is not applicable when the location of interest $x$ is close to the impact plane $\left(x<x^{*}\right)$. In this case, the peak stress can be obtained by tracing all wave interactions and it is distance-dependent. When $x>x^{*}$ in elastic regime, a mean stress $\sigma_{\text {mean }}$ exists and the first peak stress will be close to $1.274 \sigma_{\text {mean }}$. This can be deduced from the fact that the integral in Equation (13) reaches the first maximum value of about 0.94 when $B$ is approximately 2.3. From Equations (17) and (8), we can write

$$
\sigma_{\text {peak }} \approx 1.274 \sigma_{\text {mean }}=1.274 \frac{\rho_{3} c_{3} \tilde{\rho}_{0} c_{0} v_{0}}{\rho_{3} c_{3}+\tilde{\rho}_{0} c_{0}} .
$$

Consequently, the linear relationship between $\sigma_{\text {peak }}$ and $\sigma_{\text {mean }}$ ensures that factors that affect the peak also affect the mean stress in the same manner. So it is convenient as well as necessary to review the effects of heterogeneity factors on mean stress $\sigma_{\text {mean }}$.

1. The comparison between Figure 10(a) and 10(b) shows that the flyer plate with higher impedance results in higher $\sigma_{\text {mean }}$. By examining Equation (18), it can be seen that $\sigma_{\text {mean }}$ will increase with the effective impedance of the target plate (represented by $\tilde{\rho}_{0} c_{0}$ ).

2. Stacking sequence (A/B vs. B/A) does not affect $\sigma_{\text {mean }}$

3. Both $\tilde{\rho}_{0}$ and $c_{0}$ are independent of interface density and distance $x$, so the mean stress level in a given bilaminate is independent of interface density and $x$.

4. Thickness ratio $h_{1} / h_{2}$ (if $\rho_{1} c_{1}>\rho_{2} c_{2}$ ) increases $\sigma_{\text {mean }}$. The opposite is true when $\rho_{1} c_{1}<\rho_{2} c_{2}$.

Though the above analysis is strictly applicable only to elastically loaded cases, some of these observations can be extended to shock loading conditions with some modifications. We are interested in shock cases, since many of the experimental data correspond to this regime. In shock loadings, the existence of $\sigma_{\text {mean }}$ is still valid strictly from the consideration of a dynamic equilibrium state. The actual magnitude of $\sigma_{\text {mean }}$ for shock loadings can be computed using Equation (8) by appropriate modifications and this equation has already been validated ( Section 3.1.1). The ratio $\sigma_{\text {peak }} / \sigma_{\text {mean }}$ (which was found to be 1.274 for elastic case) does not remain a constant under shock loading conditions. The wave velocities depend highly nonlinearly on the stress levels in shock wave regime, and the magnitude of $\sigma_{\text {peak }}$ depends on specific factors including impact velocity and other heterogeneity factors. We can still examine the effect of heterogeneity factors on the shock response by examining previous experiments. For example, in the experimental data by Clements et al. [8] (the configuration is shown in Table 1, row 5), the particle velocity history is recorded when an epoxy and epoxy-graphite mixture layered system was subjected to a plate impact test. Fortuitously the combination of materials is such that $I \approx 0$, similar to that of a homogeneous material. Since $x^{*} \gg 0$ for such systems, the location of measurement $x \ll x^{*}$, and hence the peak stress is very close to the mean stress. On the other hand, the experimental data by Oved et al. [6] (configuration shown in Table 1, row 3) for Cu-PMMA $(I \approx 0.743)$ shows a wide oscillation with $\sigma_{\text {peak }}$ much higher than $\sigma_{\text {mean }}$. In this case, the ratio of peak stress over mean stress can be much higher than 1.274, which can be also observed from other experiments [9].

\subsection{Oscillations}

\subsubsection{Amplitude of oscillations}

From Equation (11), it is also clear that stress oscillates in the pulse duration segment and that the peaks always decay. For elastic analysis, strength of the peaks in the far field decays in a standard manner: the first peak is about 1.274 and the first valley is 0.81 , the second peak is about 1.152 and so forth. In the shock regime, amplitudes of oscillations are generally higher as the loading strength increases. The observation from past experiments as shown in Table 1 appears to suggest that the amplitudes of oscillations qualitatively decay slower than the 1.274, 1.152 pattern. Again, this is not true for systems with negligible impedance mismatch at $x<x^{*}$. Since we have examined the effects of various factors on the first peak stress in Section 3.2, the amplitude of oscillations are not extensively studied here.

Interestingly in shock response, experiments do not exhibit a constant value of mean stress. The stress tends to oscillate about a decreasing mean, or the mean stress "decreases" with time (see experiments by Oved et al. [6] and Zhuang [9]). This behavior can be due to some time-dependent dissipation mechanisms and this needs further investigation. However, it is noticed from the experimental data that the mean stress only decreases slightly. Besides, the second, the third peak and subsequent peaks are lower than the first peak. Hence, we ignore this phenomenon for the time being and define the initial mean stress of the first peak as the mean stress for the whole wave profile. When materials are under shock and sustain high steady stress, the density and wave ve- 
locity of each constituent are much higher than original values. Also, the thickness of each layer is smaller than the original value. As a result, the effective shock velocity $c_{0}$ and the mean stress $\sigma_{\text {mean }}$ are higher than the prediction using elastic analysis. However, it should be noted that for systems with very little impedance mismatch, only negligible oscillations are generated and most energy is contained in the head wave at moderate distance, when $x<x^{*}$ we can assume that $\sigma_{\text {peak }} \approx \sigma_{\text {mean }}$.

\subsubsection{Period of oscillations}

It is found that the rise time is roughly proportional to the oscillation period, so the trend in period of oscillations is similar to the trend in rise time. Figure 10 shows that the period of the oscillations varies from system to system, and system with higher impedance mismatch will result in longer rise time and lower oscillation frequency. It can be seen from Equation (13) that dimensionless factor $B$ directly determines the oscillation frequency. Again, since $B$ is obviously distance- $x$ and time- $t$ dependent, the oscillations are also distanceand time-dependent. The effects of material heterogeneity factors on the period of oscillations can be evaluated from Figure 11. Similar to the analysis of rise time, high impedance mismatch between material A and B results in long period of oscillations. Period of oscillations decrease with interface density, while thickness ratio usually has a lesser influence.

\section{Summary}

A thorough understanding of the wave structure under these loading conditions can help us in our overall goal of optimizing the composite structures. Homogenization methods cannot be used for this purpose, though they are very effective in low velocity impact designs. An analytical solution for the case of layered heterogeneous systems subjected to high velocity plate impact has been presented. Though the solution is strictly valid for elastic cases, method to extend its validity to shock regimes has been outlined. With the assumption that the interfaces are fully bonded and that the constituents are present in a damage-free state, we have identified three different heterogeneity factors that influence the material response, impedance mismatch, interface density and thickness ratio. The effect of these factors on the wave interaction and hence the observed wave structures are carefully examined. Some specific observations are as follows:

1. Complex laminated engineering composites such as 2D woven GRP can be idealized as planar layered systems to study the impact behavior, when the inherent wave interactions are significant. There is still a lack of experimental data for building a clear link between these two systems.
2. Both impact loading conditions and architecture of the composite material systems dominate the impact response.

3. Impedance mismatch governs the reflection ratio and transmission ratio at each single scattering event. It is also a predominant factor in determining the overall stress wave structure; high impedance mismatch results in "delayed arrival" of the wave at a far distance, long rise time, high oscillation amplitude and low frequency.

4. Interface density determines the number of waves with a certain propagating path in between the layers before the wave reaches a particular point. Therefore, interface density determines the strength of a wave train besides impedance mismatch. Overall, high interface density indicates a short rise time and high oscillation frequency. In shock regime, high interface density can result in shock wave "overtaking" effect, which is related to high peak stress and increased oscillation frequency.

5. Thickness ratio determines the wave propagation patterns inside the layered systems. When the thickness ratio is extremely low, the rise time is almost vertical and the oscillations do not exhibit the usual harmonic behavior. Thickness ratio directly affects the mean stress value.

6. The observed anomaly that the measured wave speed in a layered system can be lower than either of its constituents for a certain material and thickness ratio combination is explained. The effect can be understood from the fact that the wave front (elastic or shock) dies out before the measurement location and the wave front (only shock waves) slows down when propagating through more unit cells.

Acknowledgments - The authors acknowledge United States Army (DAAD19-02-1-0376) with the project monitor Dr. Bruce LaMattina. The work was motivated by Dr. A. M. Rajendran, of $\mathrm{ARO}$, and the salient features in this paper can be attributed to the many useful discussions with him.

\section{Appendix A. Extension to shock response}

For laminated systems under shock loading, it is necessary to relate shock velocity, density and volume to the particle velocity by means of equation of state (EOS). A general EOS takes the form

$$
U_{\mathrm{s}}=C_{0}+S_{1} u_{\mathrm{p}}+S_{2} u_{\mathrm{p}}^{2}+S_{3} u_{\mathrm{p}}^{3}+\cdots,
$$

where $S_{1}, S_{2}$, and $S_{3}$ are empirical parameters. $C_{0}$ is the sound velocity in a given material under zero pressure. The density under high pressure $\left(\rho_{i}^{\prime}\right)$ can no longer be approximated as the original density. It is directly re- 
lated to the loading strength represented by particle velocity $u_{\mathrm{p} i}$ :

$$
\rho_{i}^{\prime}=\frac{1}{1-\frac{u_{\mathrm{p} i}}{U_{\mathrm{si}}}} \rho_{i}, \quad i=1,2,3 .
$$

In the same way, the volume under high pressure $\left(V_{i}^{\prime}\right)$ is related to $u_{\mathrm{p} i}$ by

$$
V_{i}^{\prime}=\left(1-\frac{u_{\mathrm{p} i}}{U_{\mathrm{s} i}}\right) V_{i}, \quad i=1,2,3 .
$$

Therefore, in plate impact problem, according to the above equation, the thickness under shock loading condition $\left(h_{i}^{\prime}\right)$ will be

$$
h_{i}^{\prime}=\left(1-\frac{u_{\mathrm{p} i}}{U_{\mathrm{si}}}\right) h_{i}, \quad i=1,2 .
$$

New impedance ratio is approximately (assuming that material 2 is harder than material 1)

$$
R^{\prime}=\frac{\rho_{2}^{\prime} U_{\mathrm{s} 2}}{\rho_{1}^{\prime} U_{\mathrm{s} 1}}
$$

It can be seen from Equations (A.1), (A.2), and (A.4) that wave velocity, thickness and density for the laminates subjected to shock loading, all depend on the particle velocity, while they remain constant for elastic response. Though velocity is not continuous across a strong shock front, it can be assumed to be continuous for weak shocks which is the case considered here. Therefore, by substituting the $U_{\mathrm{si}^{\prime}} \rho_{i}^{\prime}$ and $h_{i}^{\prime}(i=1,2)$ into Equation (9), we obtain the late time velocity for shock loading condition $\left(\tilde{U}_{\mathrm{s}}\right)$ :

$$
\tilde{U}_{\mathrm{s}}=\frac{h_{1}^{\prime}+h_{2}^{\prime}}{\left[\left(\frac{h_{1}^{\prime}}{U_{\mathrm{s} 2}}\right)^{2}+\left(\frac{h_{2}^{\prime}}{U_{\mathrm{s} 2}}\right)^{2}+\left(R^{\prime}+\frac{1}{R^{\prime}}\right) \frac{h_{1}^{\prime} h_{2}^{\prime}}{U_{\mathrm{s} 1} U_{\mathrm{s} 2}}\right]^{1 / 2}} .
$$

Similarly, we obtain the mean stress level for layered systems $\sigma_{\text {mean }}^{\prime}$ by assuming that the layered systems is equivalent to the mixture with impedance $\tilde{\rho}_{0} \tilde{U}_{\mathrm{s}}$. Similar to Equation (8), we have

$$
\sigma_{\text {mean }}^{\prime}=\tilde{\rho}_{0} \tilde{U}_{\mathrm{s}} \tilde{u}_{\mathrm{p}}^{\prime}=\frac{\tilde{\rho}_{0} \tilde{U}_{\mathrm{s}} \rho_{3} U_{\mathrm{s} 3}}{\rho_{3} U_{3}+\tilde{\rho}_{0} \tilde{U}_{\mathrm{s}}} v_{0}
$$

Thus, we have obtained $\sigma^{\prime}$ mean (Equation (A.7)), the steady-state stress value for shock loading conditions analogous to $\sigma_{\text {mean }}$ for elastic loading. However, we need to be very cautious in selecting the number of steps (or wave trains) since in shock loading conditions different wave trains travel with different velocities. It is possible that a wave train can travel faster than its predecessor and may even overtake it. This phenomenon in shock loading conditions may dictate the use of more steps for capturing this effect.

For an observer at a given location, the first wave front propagates with longitudinal velocity into uncom- pressed media. Immediately behind the head wave, the high pressure $\sigma_{0}^{\prime}$ is achieved

$$
\sigma_{0}^{\prime}=\frac{\rho_{3} U_{\mathrm{s} 3} \rho_{1} U_{\mathrm{s} 1} v_{0}}{\rho_{3} U_{\mathrm{s} 3}+\rho_{1} U_{\mathrm{s} 1}} .
$$

Thus, the second wave train can travel faster than the head wave since the material is highly compressed given by Equation (A.8). As propagation distance $x$ increases, the second wave can catch up with the head wave. It can be shown that this "overtaking" effect is critical for capturing the peak stress in shock wave regime.

The steps in the analytical procedure for determining the stress response under shock loading conditions are as follows:

1. The shock velocities, $U_{\text {si }}$ and the thickness, $h_{i}^{\prime}$, should be calculated by considering EOS, as shown in Equations (A.1) - (A.4).

2. The mean stress $\sigma_{\text {mean }}^{\prime}$ should be computed using Equations (A.6) and (A.7).

3. Incident stress in the shock regime $\sigma_{0}^{\prime}$ is given by Equation (A.8). Incremental stress values at the impact plane $\Delta \sigma^{\prime}{ }_{0}, \Delta \sigma^{\prime}{ }_{1}, \ldots$ should be calculated. Modification of reflection ratio is needed based on velocity variation with pressure.

4 . The number of steps, $n$ should be carefully chosen depending on the location $x$ for which the stress is computed, $n$ should be at least equal to the number of steps needed to reach the first peak from $\sigma^{\prime}{ }_{0}$ at the impact plane.

5. The effect of 'overtaking' of a successor wave over a predecessor wave should be evaluated. When overtaking takes place, the time interval between these two waves are set to zero at the impact plane in Equation (10).

\section{References}

1. O. Sherby, S. Lee, R. Koch, T. Sumi, and J. Wolfenstine, Multilayered composites based on ultrahigh carbon steel and brass. Mater. Manufact. Process. 5 (1990), pp. 373-376.

2. H. Mahfuz, Y. Zhu, A. Haque, A. Abutalib, V. Vaidya, S. Jeelani, B. Gama, J. Gillespie, and B. Fink, Investigation of high-velocity impact on integral armor using finite element method. Int. J. Impact Eng. 242 (2000), pp. 203-217.

3. E. DeLuca, J. Prifti, W. Betheney, and S. Chow, Ballistic impact damage of s2-glass-reinforced plastic structural armor. Compos. Sci. Technol. 58 (1998), pp. 1453-1461.

4. C. Lundergan and D. Drumheller, Propagation of stress waves in a laminated plate composite. J. Appl. Phys. 422 (1971), pp. 669-675.

5. L. Barker, C. Lundergan, P. Chen, and M. Gurtin, Nonlinear viscoelasticity and the evolution of stress waves in laminated composites: a comparison of theory and experiment. J. Appl. Mech. (1974), pp. 1025-1030. 
6. Y. Oved, G. Luttwak, and Z. Rosenberg, Shock wave propagation in layered composites. J. Compos. Mater. 12 (1998), pp. 84-96.

7. G. Kanel, M. Ivanov, and A. Parshikov, Computer simulation of the heterogeneous materials response to the impact loading. Int. J. Impact Eng. 17 (1995), pp. 455-464.

8. B. Clements, J. Johnson, and R. Hixson, Stress waves in composite materials. Phys. Rev. E 54 (1996), pp. 6876-6888.

9. S. Zhuang, Shock wave propagation in periodically layered composites. PhD Dissertation, California Institute of Technology; 2002

10. S. Zhuang, G. Ravichandran, and D. Grady, An experimental investigation of shock wave propagation in periodically layered composites. J. Mech. Phys. Solids 51 (2003), pp. 245-265.

11. L. Barker, A model for stress wave propagation in composite materials. J. Appl. Mech. 5 (1971), pp. 140-162.

12. D. Dandekar and P. Beaulieu, Shots 518 and 521-1. AMD 48 (1995), pp. 63-70.

13. J. Boteler, A. Rajendran, and D. Grove, Shock wave profiles in polymer matrix composite. Shock Compress. Condensed Matter (1999), pp. 563-566.

14. C. Sve, Stress wave attenuation in composite materials. J. Appl. Mech. (1972), pp. 1151-1153.

15. C. Chen and R. Clifton, Asymptotic solutions for wave propagation in elastic and viscoelastic bilaminates, developments in mechanics. In: Proceedings of the 14th Midwestern Mechanics Conference, University of Oklahoma, vol. 8; 1975. p. 399-417

16. J. Pock and G. Gurtman, Dispersive pulse propagation parallel to the interfaces of a laminated composite. J. Appl. Mech. (1969), pp. 479-484.

17. M. El-Raheb, Transient elastic waves in finite layered media: one-dimensional analysis. J. Acoust. Soc. Am. 941 (1993), pp. 172-184.
18. M. El-Raheb, Simplified analytical models for transient uniaxial waves in a layered periodic stack. Int. J. Solids Struct. 3423 (1997), pp. 2969-2990.

19. X. Chen, N. Chandra, and A. Rajendran, Analytical solution to the plate impact problem of layered heterogeneous material systems. Int J Solid Struct, accepted

20. V. Nesterenko, Dynamic of heterogeneous materials. , Springer Verlag, Berlin (2001).

21. N. Naik and P. Shembekar, Elastic behavior of woven fabric composites: I-lamina analysis. J. Compos. Mater. 2615 (1992), pp. 2196-2225.

22. P. Shembekar and N. Naik, Elastic behavior of woven fabric composites: Ii-laminate analysis. J. Compos. Mater. 2615 (1992), pp. 2226-2246.

23. S. Chou and E. Deluca, Dynamic response of s2-glass reinforced plastic structural armor. In: ARL-SR-55, Army Research Laboratory (1993).

24. A. Khan, O. Colak, and P. Centala, Compresive failure strengths and modes of woven s2-glass reinforced polyester due to quasi-static and dynamic loading. Int. J. Plast. 18 (2002), pp. 1337-1357.

25. N. Chandra, X. Chen, and A. Rajendran, The effect of material heterogeneity on the shock response of layered systems in plate impact tests. J. Compos. Technol. Res. 244 (2002), pp. 232-238.

26. N. Chandra, X. Chen, and A. Rajendran, The shock response of layered systems in plate impact tests. In: Proceedings of the Shock Wave Processes in Condensed Matter, Edinburgh, May 19-24, 2002.

27. J. Johnson and L. Barker, Dislocation dynamics and steady plastic wave profiles in 6061-T6 aluminum. J. Appl. Phys. 40 (1969), p. 4321. 